\title{
A Biology Experimental Study on the Effect of Bionic Painless Needles on Pain Relief
}

\author{
Jingchun Wang ${ }^{1, a}$, Yu Chen ${ }^{2, b^{*}}$ and Lingxin Kong ${ }^{2,3}$ \\ ${ }^{1}$ College of Biological and Agricultural Engineering, Jilin University, China \\ ${ }^{2}$ Engineering training center, Jilin University, China \\ a13844060515@139.com, bcy2453@sina.com, ‘36193209@qq.com \\ *The corresponding author
}

Keywords: Bionic structure; Painless needle; Biology experimental study; Pain relief

\begin{abstract}
Several kinds of painless biomimetic needle structures with high drag reduction effect were obtained by means of experimental optimization design. The changes of heart rate and blood pressure as the index of pain sensitivity were selected. The experimental study on the biological function of bionic needles was carried out. Rat heart rate, blood pressure and other indicators of changes in the law of pain is found out. The pain reduction effects of several bionic painless needles are tested by the experiment. The results of the experiment had been consistent with experiment optimization design.
\end{abstract}

\section{Introduction}

Many scholars with the actual situation carry out the theoretical research and experimental study of the needle reduction and pain reduction effect from different angles, while developing a variety of painless injection methods and techniques[1、2、3]. Before this paper, professor Wang Jingchun of Jilin University, Chen Lili, Gu Songtao, Li Yan, etc. study the coupling bionic painless needle, and do the relevant drag reduction experimental study. Experimental results show that the corrugated type painless needles have the best drag reduction effect, with a maximum drag reduction rate of $51.13 \%$.

However, it is required to measure and quantitatively analyze the pain produced by painless acupuncture in order to further demonstrate the analgesic effect of the painless needle. Although the drag reduction mechanism of the groove-type bionic needle is further analyzed by Qi Yingchun et al., similar experiments have not been carried out in vivo [4]. The needle itself, however, ultimately needs to be applied to the organism.

The experiment is carried out on the application of bionic needles in medicine which shows the relationship between drag reduction effect and pain reduction effect. The relevant physiological indicators are determined as the standard test of pain. This study provides theoretical guidance and technical support for the application of bionic painless needles in medical field. Theoretical guidance and technical support are provided to the application of bionic painless needle in medical field. Associated morphology and parameters of painless needle are obtained which has the optimal pain relief effect of the painless needle.

\section{Structural Optimization of Bionic Painless}

A lot of practice and research results show that the application of non-smooth surface structure can effectively reduce the friction between objects. According to this theory, Professor Wang Jingchun studied the bionic needles, and designed bionic needles with corrugated structure, sawtooth structure, pit structure and groove structure [5]. The design of the bionic needle is based on medical 6 disposable syringe needle design improvement which widely used clinically. The groove type bionic needle is different from other unit structures which are machined in accordance with the longitudinal groove design [6]. 
By summarizing the previous experiments, several kinds of bionic painless surface structures with the best drag reduction effect are obtained, which mainly includes a corrugated needle and a grooved needle [7]. The drag reduction effect of needles is satisfactory according to the test conclusions. Table 1 summarizes the best obtained several types of needle parameters.

Table 1 Bionic needle shape and structure parameters

\begin{tabular}{ccccc}
\hline Group Number & Form[mm] & Depth[mm] & Spacing[mm] & Width[mm] \\
\hline 1 & Corrugated Type & 0.050 & 0.75 & 0.30 \\
2 & Corrugated Type & 0.080 & 0.75 & 0.30 \\
3 & Corrugated Type & 0.050 & 0.75 & 0.20 \\
4 & Groove Type & 0.05 & $\mathrm{n}=15$ & 0.03 \\
\hline
\end{tabular}

In four different types of needles, No.1 and No. 2 needles are the two groups of needles with the smallest frictional resistance in the friction resistance experiment. No.3 needle is the optimal combination in orthogonal test results of needle drag reduction test. No. 4 needle is the groove structure of the optimization results of bionic needles in the comprehensive consideration of needle strength and needle size $[8,9,10]$.

\section{Biology Experimental Study on Pain Relief}

In order to determine whether the needles with a good drag reduction effect has a good effect of reducing pain, the effect of the bionic painless needle in reducing pain is further explored by acupuncture on the living organism. According to the experimental scheme, a series of physiological indexes are measured during the experiment. The rats are preliminarily tested in order to determine the influence of acupuncture on the pain and the change trend of each index. Then the rats are subjected to a systematic acupuncture test.

Pre-Experiment Process and Analysis. The mean values and average values of heart rate, diastolic blood pressure, systolic blood pressure and mean pressure are obtained by analyzing the results of eight measurements by means of multiple measurements. The changes of blood pressure and heart rate after acupuncture are observed and recorded. The above experiment can be judged initially. Table 2 is the specific data.

Table 2 Pre-experiment values of Heart rate and blood pressure values

\begin{tabular}{cccc}
\hline Animal Number & Test number & HR [Times / minute] & BP [mmHg] \\
\hline 1 & 1 & 420 & 136 \\
1 & 2 & 425 & 141 \\
1 & 3 & 416 & 142 \\
1 & 4 & 426 & 142 \\
1 & 5 & 418 & 139 \\
1 & 6 & 418 & 139 \\
1 & 7 & 405 & 144 \\
1 & 8 & 429 & 132 \\
Mean Value & & 419.625 & 139.375 \\
1 & Acupuncture 1 & 458 & 152 \\
1 & Acupuncture 2 & 480 & 133 \\
1 & Acupuncture 3 & 447 & 170 \\
\hline
\end{tabular}


The universality and reliability of large sample are lacking in this experiment. However, this pre-experiment gives a clear direction, which is that the rat's physiological indicators will be some changes in the course of pain.

Acupuncture Test. To test the pain reduction effects of several different needles, 64 rats are used and divided into 8 groups as subjects. Eight rats are randomized into one group. Rats in each group are given habitual tail training for one week before each test to ensure that rats are adapted to the set of tail method.

In the 8 experimental groups, Groups 1 to 4 are used as the basic group, and all rats are treated with ordinary needles. Groups 5 to 8 are used as the control group, and each kind of bionic needle was used for acupuncture. The grouping of the bionic needles is shown in Table 3.

Table 3 The grouping of the bionic needles

\begin{tabular}{ccccc}
\hline Group Number & Form[mm] & Depth[mm] & Spacing[mm] & Width[mm] \\
\hline 5 & Corrugated Type & 0.05 & 0.75 & 0.2 \\
6 & Groove Type & 0.05 & $\mathrm{n}=15$ & 0.03 \\
7 & Corrugated Type & 0.05 & 0.75 & 0.3 \\
8 & Corrugated Type & 0.08 & 0.75 & 0.3 \\
\hline
\end{tabular}

The above eight groups of experimental results are observed that the heart rate and blood pressure of the rats are changed before and after acupuncture, and the change trend is consistent. The changes of heart rate and blood pressure in each group are relatively consistent.

The changes of heart rate and blood pressure of rats in each group when receiving different needles acupuncture are shown in Table 4 and Table 5. The values of each group are the average of the rats' heart rate and blood pressure. Groups $1 \sim 4$ are the average of heart rate and blood pressure after acupuncture with ordinary needles, and the changes of heart rate and blood pressure are different in different acupuncture groups.

Table 4 Changes of heart rate in different needles

\begin{tabular}{ccc}
\hline Group & HR before acupuncture[ c. p. m.] & HR after acupuncture[ c. p. m.] \\
\hline $1-4$ & 363.47 & 485.31 \\
5 & 338.88 & 428.5 \\
6 & 399.38 & 492.25 \\
7 & 358.38 & 426.5 \\
8 & 372 & 438.13 \\
\hline
\end{tabular}

Table 5 Changes of blood pressure in different needles

\begin{tabular}{ccc}
\hline 组别 & BP before acupuncture $[\mathrm{mmHg}]$ & BP before acupuncture $[\mathrm{mmHg}]$ \\
\hline $1-4$ & 90.53 & 185.06 \\
5 & 92.63 & 164.25 \\
6 & 103.75 & 180.5 \\
7 & 90.38 & 145.5 \\
8 & 98.88 & 148.38 \\
\hline
\end{tabular}

Analysis of Result. To compare the test data in detail, we divided the eight groups into four control groups: group A (group 1 and group 5), group B (group 2 and group 6), group C (group 3 
and group 7) (Group 4 and group 9).Then the data from the four control groups are compared and analyzed.

Data analysis within each control group the changes of blood pressure and heart rate before and after acupuncture in each control group were recorded as $\Delta \mathrm{HR}$ and $\triangle \mathrm{BP}$, and the change rule was observed. Table 6 shows the change of heart rate and blood pressure in each control group after data processing.

Table 6 Changes of heart rate and blood pressure in different control groups

\begin{tabular}{ccc}
\hline Group & $\Delta$ HR [ c. p. m.] & $\Delta$ SBP [mmHg] \\
\hline Group A & 33.5 & 19.875 \\
Group B & 27.375 & 12.625 \\
Group C & 53.875 & 45.875 \\
Group D & 55.875 & 46.75 \\
\hline
\end{tabular}

By comparing the heart rate and blood pressure extent of changes can reflect the degree of bionic needles than the average needle has a certain pain reduction effect, and it can be compared that in several kinds of bionic needles, which needle causes the smallest change. Specific comparison are shown in Fig. 3.

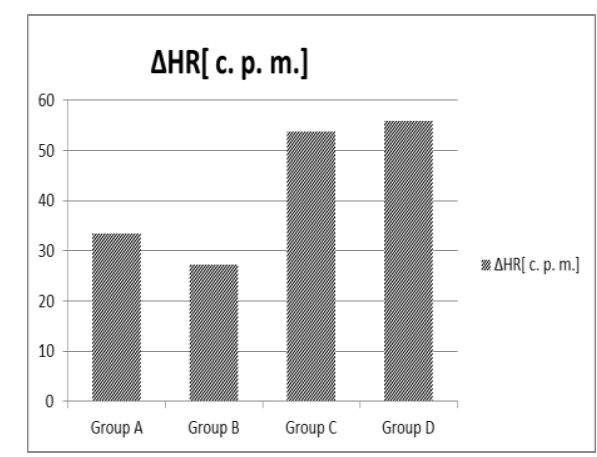

(a)

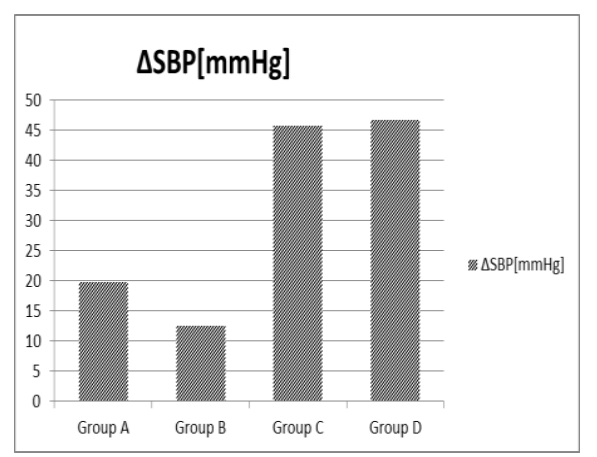

(b)

Figure 1. Comparison of Heart Rate and Blood Pressure Change in Four

As can be seen from Fig. 1, four groups of comparative tests verify that the four kinds of bionic needles for the pain reduction in rats have played a relatively significant role. The change of heart rate and blood pressure in group $\mathrm{C}$ and group $\mathrm{D}$ are significantly higher than those in the first two groups, which indicated that the two kinds of bionic painless needles have better effect of reducing pain. The size of non-smooth unit of the needle in the group 8 corresponding to group $\mathrm{D}$ is bionic needle with depth $0.08 \mathrm{~mm}$, spacing $0.75 \mathrm{~mm}$ and width $0.3 \mathrm{~mm}$, and the bionic needle with the largest drag reduction rate was measured.

Data analysis between each control group In order to reflect the comprehensive nature of the experimental data, the data need to be analyzed from other angles, and it is proved by the analysis that the above conclusions are satisfied.

The data are compared with, which are the extent of changes of heart rate and blood pressure among group5 to 8 and all other rats after acupuncture with general acupuncture.

It can be seen from the graph 2 that when the heart rate data of 5 to 8 groups are compared in particular with the average heart rate of all 32 basal rats (all rats receiving general needle acupuncture), and the results are in agreement with the previous conclusions match. As shown, group 7 and group 8 have the smallest change of heart rate before and after acupuncture, and change of group 8 is smaller than change of group 7. Among the four groups of rats treated with bionic needles, group 6 had the greatest change in heart rate. 


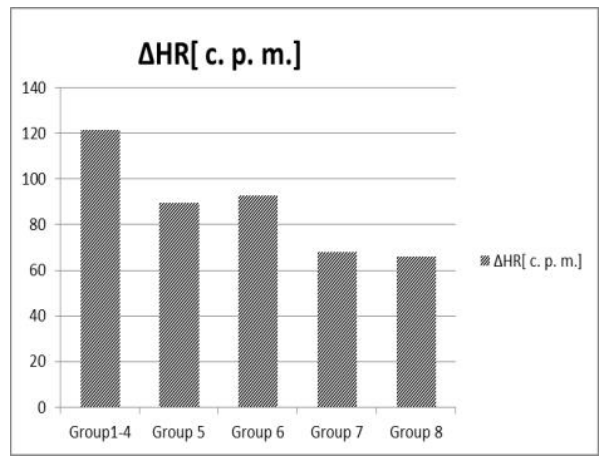

Figure 2. Changes of heart rate in rats induced by various bionic needles

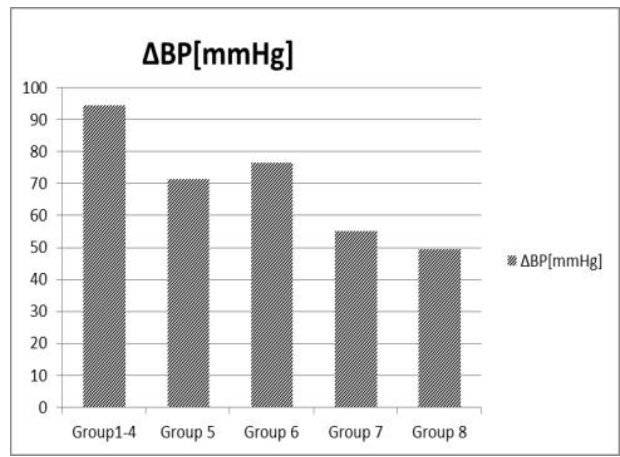

Figure 3. Changes of blood pressure in rats induced by various

By the same token, the change of blood pressure caused by various bionic needles was compared with the change of blood pressure of all rats after acupuncture by general needle, as shown in Fig. 3 .

As can be seen from the figure, the amount of blood pressure change in Group 8 is still the smallest of the four groups of bionic needles after acupuncture, while the change in group 6 is the largest, which is reflected in Fig. 4 shows the size of heart rate changes in the amount of order consistent. It shows that the results of the experiment can be mutually verified, and also shows that the various bionic needle acupuncture effect has its particularity and regularity.

\section{Conclusions}

Tests have shown that there is a significant change in heart rate and blood pressure during pain in rats. When the rats feel pain, heart rate, blood pressure values are on the rise.

Bionic needles do have some effect on reducing pain in rats when they are intramuscularly injected.

Bionic needles with different structures have different effects on pain caused by acupuncture. Which caused the heart rate and blood pressure changes in the smallest, should be the best pain reduction effect of a bionic needle. The optimal structure of the needle structure is corrugated structure: depth $0.08 \mathrm{~mm}$, spacing $0.75 \mathrm{~mm}$, width $0.3 \mathrm{~mm}$.

\section{Acknowledgements}

Key project of Jilin province science and technology development, number: 20120448 China

\section{References}

[1] Roxhed, N. Painless Drug Delivery Through Microneedle-Based Transdermal Patches Featuring Active Infusion. Biomedical Engineering, March 2008, p1063-1071.

[2] Mark R Prausnitz. Microneedles for transdermal drug delivery. Vol 56, Issue 5, 27 March (2004), p581.

[3] Y.H. Gao. Micro needle transdermal drug delivery system research progress. External treatment of traditional Chinese medicine, 2005, 14 (3): p3. (In Chinese)

[4] Y.C. Qi, Q.Cong, J.Y. Wang, Xin QI. Optimization Design and Drag Reduction Mechanism Research on Groove Shape Bionic Needle. Journal of mechanical industry, (2012)8 (15) : p126. (In Chinese)

[5] J.C.Wang, L.L.Chen, L.Q. Ren, S.T. Gu, Qian Cong Experimental research on drag reduction of bionic injector needles Journal of Jilin University:Eng and Technol Ed. Vol 38(2008)No.2,p.379. (In Chinese) 
[6] Yan Li The Research on the Characteristics of Bionic Needle's Puncture Resistance (MS., Jilin University,China 2011) (In Chinese)

[7] J.C. Wang Coupling Bionic Research on Painless Injector Needles Based on Insect Piercing-sucking Mouthpart (Ph.D., Jilin University,China 2008) (In Chinese)

[8] Yu Chen. Experimental Study about the Performance of Bionic Painless Needle and the Associated Biology Experiment (MS, Jilin University,China 2012). (In Chinese)

[9] Schatzmann, U.Spadavecchia, C. Significance, diagnosis and interpretation of pain. Pferdeheilkunde, Vol.20.(2004).No.1 p.51.

[10]CB Johnson. New Approaches to Identifying and Measuring Pain. OIE Technical Series, (2008).p1. 